\title{
OB-stars as extreme condition test beds
}

\author{
Joachim Puls ${ }^{1}$, Jon O. Sundqvist ${ }^{1}$ and Jorge G. Rivero González ${ }^{1}$ \\ ${ }^{1}$ Universitätssternwarte der Ludwig-Maximilians-Universität München \\ Scheinerstr. 1, D-81679 München, Germany \\ email: uh101aw@usm.uni-muenchen.de (J.P.)
}

\begin{abstract}
Massive stars are inherently extreme objects, in terms of radiation, mass loss, rotation, and sometimes also magnetic fields. Concentrating on a (personally biased) subset of processes related to pulsations, rapid rotation and its interplay with mass-loss, and the bistability mechanism, we will discuss how active (and normal) OB stars can serve as appropriate laboratories to provide further clues.
\end{abstract}

Keywords. hydrodynamics, instabilities, line: formation, stars: abundances, stars: early-type, stars: evolution, stars: mass loss, stars: oscillations, stars: rotation, stars: winds, outflows

\section{Introduction}

Massive stars are inherently extreme objects, in terms of radiation, mass loss, rotation and sometimes also magnetic fields. Thus, they can serve as test beds for extreme conditions and corresponding theoretical predictions. Such tests are, e.g., particularly important for our understanding of the (very massive) First Stars and for the physics of fast rotation in massive stars, which is a key ingredient in the collapsar model of long Gamma Ray Bursts. In this review we discuss how a variety of physical processes present in massive stars can affect both their stellar photospheres and/or winds, and how active (and normal) OB stars can be, and are, used as appropriate laboratories to provide further clues. In the following, we concentrate on a (personally biased) subset of processes related to pulsations (Sect. 2), rapid rotation and its interplay with mass-loss (Sect. 3), and mass-loss itself, particularly on the bi-stability mechanism (Sect. 4).

\section{Pulsations}

\subsection{Pulsating B-supergiants}

Well outside the instability strips of $\beta$ Cep and slowly pulsating B-stars (SPB), Waelkens et al. (1998) via HIPPARCOS detected 29 periodically variable B-supergiants. A corresponding instability region had not been predicted at that time. Meanwhile, however, Pamyatnykh (1999) and Saio et al. (2006, see also this volume) identified such regions for pre-TAMS and post-TAMs objects, respectively, with SPB-type of oscillations (high order g-modes). These regions are indicated in Fig. 1, together with results from quantitative spectroscopy by Lefever et al. (2007), for those of the above 29 supergiants with sufficient spectral information. Obviously, most of these objects are located very close to the high gravity limit of the predicted pre-TAMS or within the predicted post-TAMS instability strips for evolved stars. Together with their multi-periodic behaviour, this strongly suggests that these objects are opacity-driven non-radial pulsators (NRPs), and thus are ideal test beds for asteroseismologic studies of evolved massive stars. Note that Lefever et al. (2007) found additional periodically variable objects not known to be pulsators so far, and suggested, from their pulsational behaviour and their positions, that these objects are g-mode pulsators as well. Two of them, HD 64760 (B0.5 Ib) and 


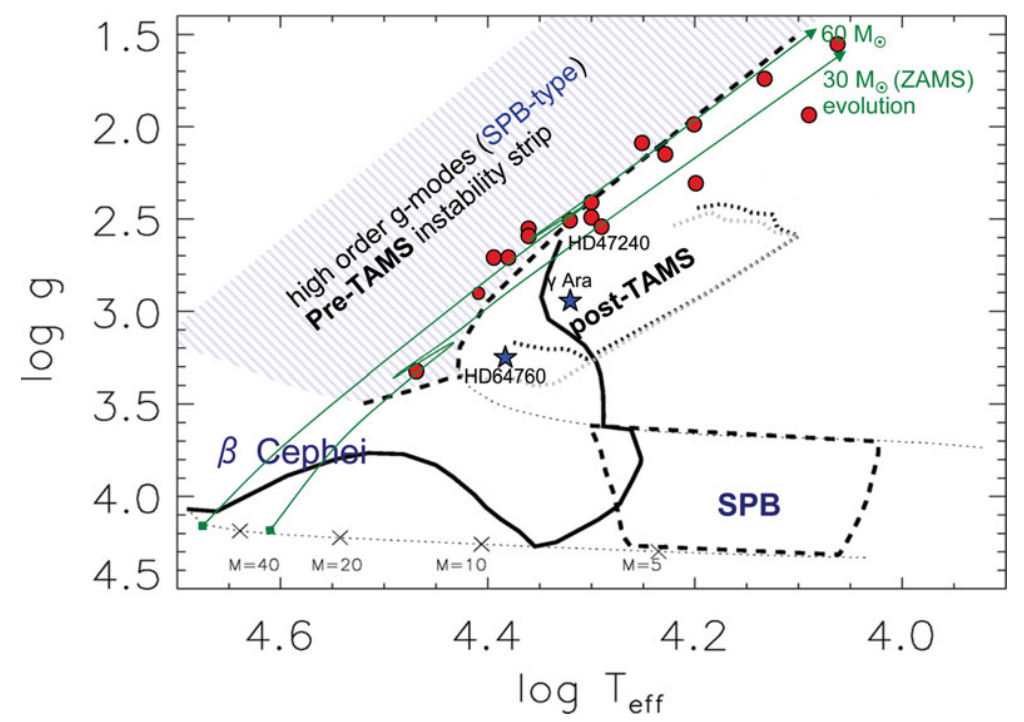

Figure 1. $\log T_{\text {eff }}-\log g$ diagram for hot massive stars. Indicated are the instability regions for $\beta$ Cep stars (solid bold), SPBs close to the ZAMS (dashed bold) and SPBs of evolved type as predicted by Pamyatnykh (1999) (pre-TAMS, blue hatched) and Saio et al. (2006) (post-TAMS, dotted). Red dots correspond to the positions of slowly pulsating B-supergiants from the sample by Waelkens et al. (1998) as derived by Lefever et al. (2007), and blue asterisks are two newly suggested g-mode pulsators from the same work. See text for further details. Adapted from Lefever et al. (2007).

$\gamma$ Ara (= HD 157246, B1 Ib), are explicitly indicated in Fig. 1, together with HD 47240 (B1 Ib) from the original sample by Waelkens et al., and will be referred to later on.

\subsection{Macroturbulence}

A present key problem in atmospheric diagnostics by high resolution spectroscopy is the finding that the line-profiles from (at least) late O- and B-supergiants display substantial extra-broadening (in addition to the well-known effects from rotation etc.), which has been termed 'macro-turbulence' (for details and references, see Simón-Díaz et al. 2010). This extra-broadening can be simulated by allowing for a supersonic Gaussian or quasi-Gaussian velocity distribution in photospheric regions, which is difficult to justify physically. Recently, however, Aerts et al. (2009) showed that such extra-broadening can be reproduced from the collective effect of low-amplitude g-mode oscillations. First hints that this scenario might be realistic have been found by Simón-Díaz et al. (2010), from a tight observed correlation between the peak-to-peak amplitudes of velocity moments measured from (variable) photospheric profiles of B-supergiants and the derived macroturbulent broadening. Given the ubiquity of macro-turbulence in hot massive stars, this tentatively suggests that a large fraction of OB-stars are non-radial pulsators (see also Fullerton, this volume).

\subsection{Triggering of structure/clump formation}

With respect to their stellar winds, pulsations in massive stars might be responsible for inducing large-scale structures, such as co-rotating interaction regions (CIRs, see Fullerton, this volume), and, particularly, for triggering the formation of clumps: To reproduce the observed X-ray emission from hot stellar winds, $L_{\mathrm{x}} \approx 10^{-7} L_{\mathrm{bol}}$, the linedriven (or deshadowing) instability related to radiative line driving needs to be excited by deep-seated photospheric disturbances of a multitude of frequencies (NRPs?), giving 
rise to strong clump-clump collisions and consequently strong shocks (Feldmeier et al. 1997a,b). For models with self-excited instability alone, the predicted X-ray emission is much too weak. Moreover, such perturbations might be responsible for triggering the on-set of deep-seated wind-clumping (Sect. 4), as implied from various diagnostics (e.g., Bouret et al. 2005; Puls et al. 2006; Sundqvist et al. 2011).

\subsection{Strange mode oscillations}

In addition to 'conventional' pulsations, another class of quasi-periodic, dynamical instabilities are predicted to occur in the envelopes of luminous stars with large $L / M>10^{3}$. These are the so-called strange-mode oscillations (for details and references, see Saio and Chené, this volume), which should be particularly strong in WR-stars and might even help to initiate their winds (e.g., Wende et al. 2008). So far, there is no direct evidence of these predictions, though the strongest amplitudes of optical lpv in O-stars are located within the region of predicted strange mode oscillations (Fullerton et al. 1996), and at least for one WR star such oscillations might actually have been observed (see Chené, this volume). Alternative test beds to check the reality of strange mode oscillations might be late B-/early A-supergiants (as suggested by Puls, Glatzel, \& Aerts as targets for the micro-satellite BRITE), since, in comparison to WRs, these objects have less dense winds and 'convenient' frequencies (on the order of a few to tens of days), with predicted amplitudes of $0.1 \mathrm{mag}$ (W. Glatzel, priv. comm.). Indeed, the COROT observations of the late B-supergiant HD 50064 (Aerts et al. 2010) showed a period of 37 days, with a sudden amplitude change by a factor of 1.6. Together with other evidence (variable $\dot{M}$ etc.), Aerts et al. tentatively interpreted this finding as the result of a strange mode oscillation.

\section{Rapid rotation}

\subsection{Photospheric deformation and gravity darkening}

Rapid rotation affects the stellar photosphere in (at least) two ways. First, it becomes deformed, with $R_{\text {eq }} / R_{\text {pole }}=1.5$ at critical rotation (using a Roche model with point mass distribution, see Zhao, this volume, and Cranmer \& Owocki 1995 for details and references). The first observational test bed which confirmed the basic effect was the brightest Be star known, Achernar $=\alpha$ Eri (VLTI observations by Domiciano de Souza et al. 2003).

The second effect is gravity darkening, first suggested by von Zeipel (1924), who assumed rotational laws that can be derived from a potential, e.g., uniform or cylindrical. An important extension was provided by Maeder (1999), who considered the more realistic case of shellular rotation in radiative envelopes, where the angular velocity is assumed to be constant on horizontal surfaces (Zahn 1992). In result, the photospheric flux is proportional to the effective gravity, $\vec{F} \propto \vec{g}_{\text {eff }}(1+\zeta(\vartheta))$, with $|\zeta(\vartheta)|<0.1$ in most cases and $\zeta=0$ in the original von Zeipel case. The effective gravity is the vector sum of gravitational and centrifugal acceleration, $\vec{g}_{\text {eff }}=\vec{g}_{\text {grav }}+\vec{g}_{\text {cent }}$, i.e., lower at the equator than at the pole, with $\vec{g}_{\text {eff }}$ (pole) $=\vec{g}_{\text {grav }}$. Note that here $\vec{g}_{\text {eff }}$ is independent of the radiative acceleration! Neglecting $\zeta(\vartheta)$, for radiative envelopes we obtain $T_{\text {eff }}(\vartheta) \propto g_{\text {eff }, \perp}^{1 / 4}$, i.e., $T_{\text {eff }}$ decreases towards the equator, in dependence of the normal component of $\vec{g}_{\text {eff }}$.

Both effects are demonstrated in Fig. 2, for a typical O-supergiant rotating close and very close to critical rotation. Deformation and gravity darkening become significant only for rotational speeds higher than roughly $70 \%$ of the critical one! Test beds to check 

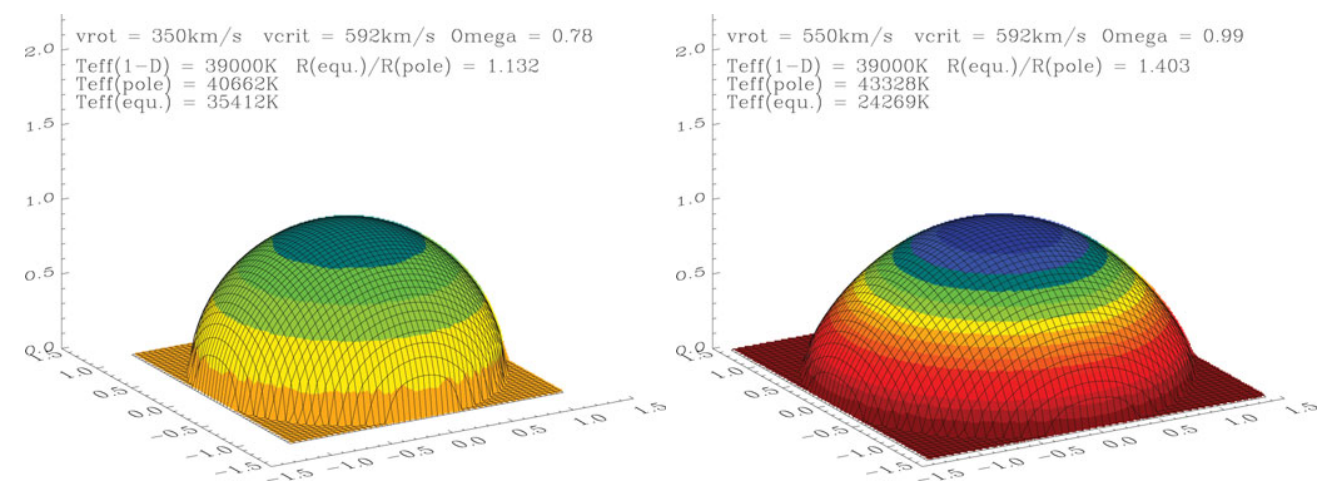

Figure 2. Predicted photospheric deformation and gravity darkening for a star similar to $\zeta$ Pup (1-D values: $T_{\text {eff }}=39 \mathrm{kK}, R_{*}=19 R_{\odot}, \log g=3.6$ ) but rotating with $78 \%$ (left) and $99 \%$ (right) of its critical angular velocity. Both figures are on the same scale, with identical color coding for $T_{\text {eff }}(\vartheta)$. The ratios $R_{\text {eq }} / R_{\text {pole }}$ and the effective temperatures at the hotter pole and cooler equator are indicated within the figures.

both effects are discussed by Zhao (this volume; see also the summary provided by van Belle 2010).

\subsection{Rapid rotation and winds}

Standard line-driven wind theory (for a recent review and references, see Puls et al. 2008) predicts that the mass-loss rate of a non (slowly) rotating star scales as

$$
\dot{M} \propto\left(N_{\text {eff }} L\right)^{1 / \alpha}\left(g_{\text {grav }} R_{\star}^{2}(1-\Gamma)\right)^{1-1 / \alpha},
$$

with $N_{\text {eff }}$ the effective number of driving lines (proportional to the force-multiplier parameter $k$, Castor et al. 1975, CAK), CAK parameter $\alpha$ (corresponding to the steepness of the line-strength distribution function), and Eddington factor $\Gamma$. Accounting for rotation (and $\Gamma$ not too large), we find that the mass-loss rate depends on co-latitude $\theta$,

$$
\begin{array}{rll}
\dot{M}(\theta) & \propto & \left(N_{\text {eff }}(\theta) F(\theta) R_{\star}^{2}(\theta)\right)^{1 / \alpha(\theta)}\left(g_{\text {eff }}(\theta) R_{\star}^{2}(\theta)(1-\Gamma)\right)^{1-1 / \alpha(\theta)} \\
& \propto \\
\text { von Zeipel } & \left(N_{\text {eff }}(\theta)\right)^{1 / \alpha(\theta)} g_{\text {eff }}(\theta) R_{\star}^{2}(\theta)
\end{array}
$$

(cf. Owocki et al. 1998). This expression renders two possibilities. i) If the ionization equilibrium is rather constant w.r.t. $\theta$ (as it is the case for O-stars), we obtain a prolate wind structure, since $g_{\text {eff }}(\theta)$ is largest at the pole. This is the $g_{\text {eff }}$-effect, see Owocki et al (1998); Maeder (1999); Maeder \& Meynet (2000). ii) If, on the other hand, the ionization equilibrium were strongly dependent on $\theta$, this would imply an oblate wind structure if the increase of $N_{\text {eff }}$ and the decrease of $\alpha$ towards the equator (as a consequence of decreasing ionization) could overcompensate the decrease of $g_{\text {eff }}$. Such a situation (the $\kappa$-effect, see Maeder 1999; Maeder \& Meynet 2000) might occur in B-supergiants (but see Sect. 4). Note, however, that no thin disk can be formed by this process alone. Note also that self-consistent 2-D hydro/NLTE calculations (though somewhat simplified) for rapidly rotating B-stars around $T_{\text {eff }}=20 \mathrm{kK}$ (i.e, just in the region where the $\kappa$ effect might be expected) by Petrenz \& Puls (2000) still resulted in a prolate wind structure, since the ionization effects turned out to be only moderate.

Of course, these predictions need to be checked observationally, particularly when considering their importance regarding stellar evolution (e.g., a pronounced polar mass loss would lead to less loss of angular momentum), and with respect to mass-loss 

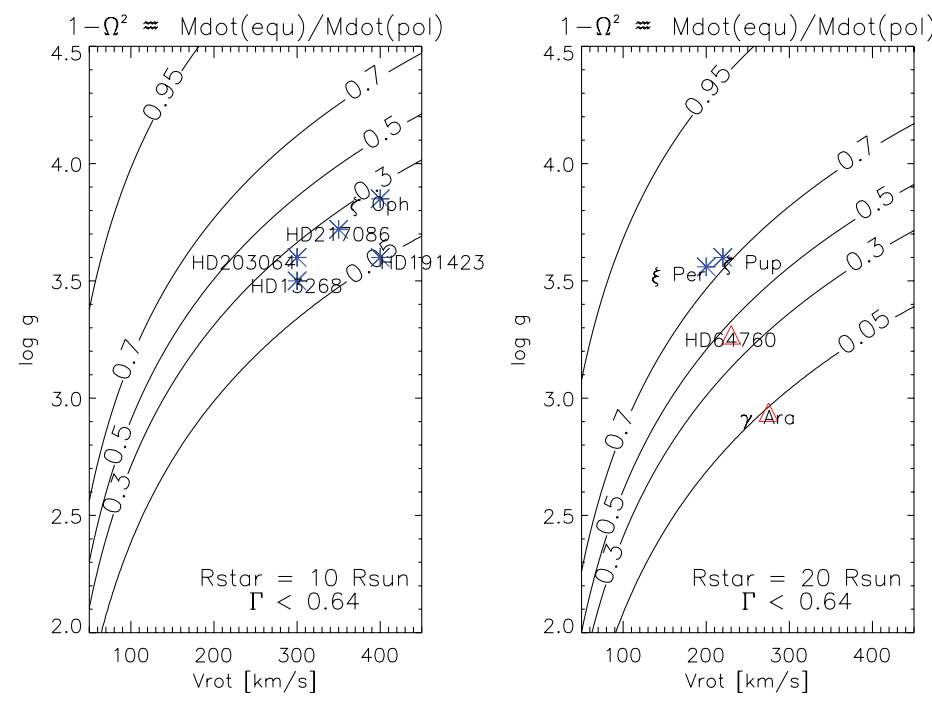

Figure 3. Iso-contours of $1-\Omega^{2} \approx \dot{M}($ eq $) / \dot{M}$ (pole) as a function of $v_{\text {rot }}$ and $\log g$, for typical dwarfs (left) and supergiants (right). Overplotted are the positions of some rapidly rotating Galactic O-stars (asterisks) and B-supergiants (triangles), assuming a minimum $v_{\text {rot }}=v \sin i$.

diagnostics (when do we need 2-D models?). To our knowledge, clear observational evidence for aspherical winds is still missing. $\dagger$ So, what are potential test beds?

In Fig. 3 we have plotted theoretical iso-contours of $1-\Omega^{2} \approx \dot{M}($ eq $) / \dot{M}$ (pole) (from Eq. 3.1 and assuming $N_{\text {eff }}$ and $\alpha$ to be constant; $g_{\text {eff }} \approx g_{\text {grav }}\left(1-\Omega^{2}\right)$ and $\left.\Omega=\omega / \omega_{\text {crit }}\right)$ as a function of $v_{\text {rot }}$ and $\log g$, for typical dwarfs (left) and supergiants (right). Overplotted are the locations of well-known Galactic rapid rotators, for a minimum value of $v_{\text {rot }}=$ $v \sin i$ (data from Repolust et al. 2004 and Lefever et al. 2007). Asterisks denote O-stars, triangles B-supergiants. All O-dwarfs/giants (left) are predicted to have a significant mass-loss contrast, below 0.3. Unfortunately, their (average) mass-loss rate is too low to lead to substantial effects in the optical wind-lines and the IR continuum, though UVspectra should be affected by deviations from spherical symmetry. For the fast rotating O-supergiants, on the other hand, the predicted effect is rather small, so nothing might be visible. Vink et al. (2009), using linear $\mathrm{H}_{\alpha}$ spectro-polarimetry, conclude that most winds from rapidly rotating $\mathrm{O}$-stars are spherically symmetric. For the two rapidly rotating Bsupergiants, HD 64760 and particularly $\gamma$ Ara, the situation is more promising, and they might be used as test beds to check the impact of rotation on the global wind topology. Remember that HD 64760 (see also Sect. 2) is one of the best studied objects in the UV (thanks to the IUE mega-campaign, Massa et al. 1995) - with the detection of CIRs and 'PAMS' (see Fullerton, this volume), both presumably related to its non-radial pulsations -, and has also been studied in the optical to clarify the interaction between NRPs and CIRs (Kaufer et al. 2006).

Fig. 4 (left) displays the corresponding $\mathrm{H}_{\alpha}$ line profiles, for the above two rapidly rotating B-supergiants and for HD 47240 (see also Sect. 2), with a somewhat lower $v \sin i$. At first glance, these profiles might indicate the presence of a disk or an oblate wind (e.g.,

$\dagger$ The polar wind structures claimed for the Be-stars Achernar (Kervella \& Domiciano de Souza 2006) and $\alpha$ Ara (Meilland et al. 2007) from NIR interferometry still need to be confirmed, given that - as discussed during this conference - for such low mass-loss rates the IR-photosphere is very close to the optical one (in other words, the IR-excess from the wind is very low). 

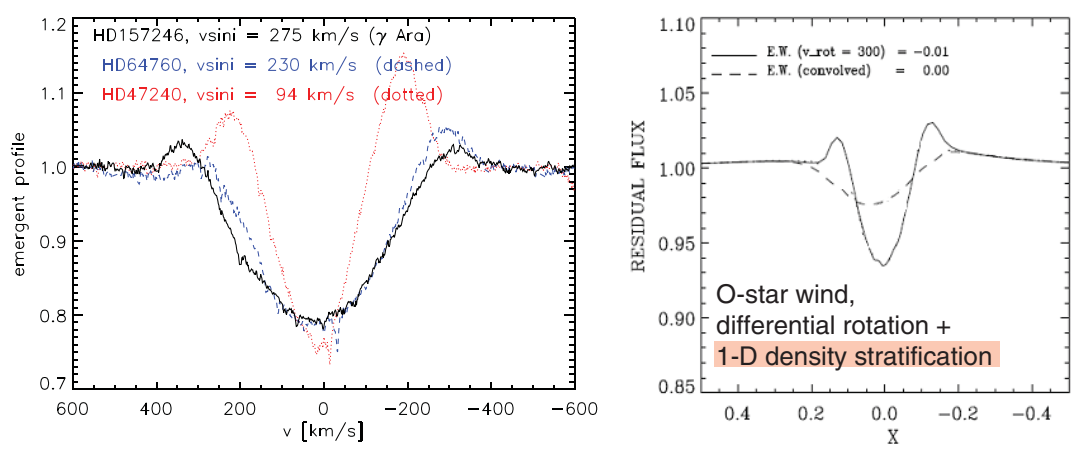

Figure 4. Left: $\mathrm{H}_{\alpha}$ line profiles for three rapidly rotating B-supergiants (spectra and data from Lefever et al. 2007). Right: Theoretical $\mathrm{H}_{\alpha}$ line profiles, for an O-star wind with spherical density stratification. Dashed: Profile convolved with a rotation profile of width $300 \mathrm{~km} \mathrm{~s}^{-1}$. Solid: 2-D line transfer allowing for differential rotation, $v_{\text {rot }} \propto 1 / r$. Adapted from Petrenz \& Puls (1996).

the $\kappa$-effect from above), but this is not necessarily the case. As shown in Fig. 4 (right), even a spherical wind can give rise to double-peaked profiles, when accounting for the wind's differential rotation (due to the so-called resonance-zone effect, Petrenz \& Puls 1996). Note that in this case the profile only depends on the product $v \sin i$ and not on the individual factors. For a 2-D density stratification, however, the profiles will look different for a prolate or oblate topology, and will depend on the individual values of $v_{\text {rot }}$ and $\sin i$ as well, which might induce a certain dichotomy. Interestingly, UV spectroscopy (via IUE) of $\gamma$ Ara by Prinja et al. (1997) gave indications for a prolate geometry, mainly because of missing or weak emission peaks in the P Cygni profiles.

\subsection{The $\Omega \Gamma$ limit}

An interesting question is what happens if a star is rapidly rotating and close to the Eddington limit. After a controversial discussion (Langer et al. 1997; Glatzel 1998), Maeder \& Meynet (2000) were able to solve this problem in an elegant way. For the following discussion, it is only important to note that the total acceleration due to gravity, centrifugal forces, and radiation pressure gradients can be expressed as $\overrightarrow{g_{\text {tot }}}=\overrightarrow{g_{\text {eff }}}\left(1-\Gamma_{\Omega}\right)$, where the effective gravity remains defined as previously, and $\Gamma_{\Omega} / \Gamma>1$ is a function of $v_{\text {rot }} / v_{\text {crit }}$. Consequently, the total acceleration can become zero before the nominal Eddington limit is reached, and this new limit is called the $\Omega \Gamma$ limit. As shown by Maeder \& Meynet (2000), the combination of rapid rotation and large $\Gamma$ can affect the total (polar-angle integrated) mass-loss rate from a radiation driven wind considerably,

$$
\frac{\dot{M} \text { (rotating) }}{\dot{M} \text { (non-rotating) }} \approx\left(\frac{1-\Gamma}{\Gamma / \Gamma_{\Omega}-\Gamma}\right)^{\frac{1}{\alpha}-1} \begin{cases}=O(1) & \text { for not too fast rotation and low } \Gamma \\ \gg 1 & \text { for fast rotation and considerable } \Gamma \\ \text { but: } & \text { max. } \dot{M} \text { limited because } L \text { limited }\end{cases}
$$

since $\alpha$ is on the order of $0.4 \ldots 0.6$. To identify potential test beds to check this important prediction, in Fig. 5 we have plotted the iso-contours of the $v_{\text {rot }}$ required for a significantly increased mass-loss rate, as a function of $T_{\text {eff }}$ and $\log g$. (A factor of four compared to the non-rotating case was chosen to allow for an easy observational check.) The red shaded region comprises the approximate locations of Galactic OB-supergiants. Overplotted are the positions of some rapidly rotating supergiants, O-types (asterisks) and B-types (triangles). The numbers in brackets are the observed $v \sin i$. Again, Osupergiants are not suited as test beds, since they would need to rotate much faster than 


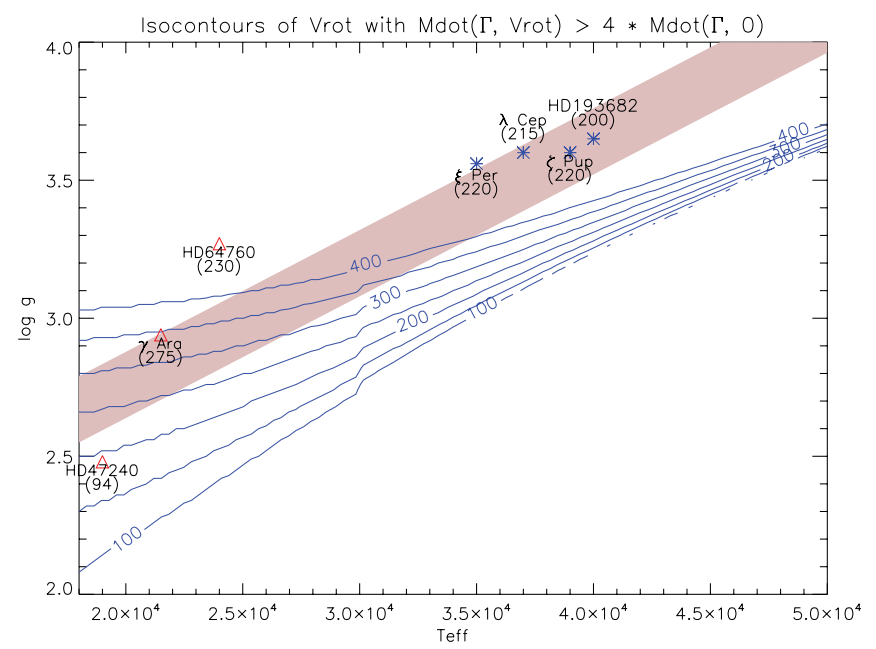

Figure 5. Iso-contours of $v_{\text {rot }}$ for which the total mass-loss rate is predicted to become increased by a factor of four, compared to the non-rotating case, as a function of $T_{\text {eff }}$ and $\log g$, together with the positions and $v \sin i$-values of some rapidly rotating $\mathrm{O}$ - and B-supergiants (asterisks and triangles, respectively). See text.

$400 \mathrm{~km} \mathrm{~s}^{-1}$ to show the required increase in $\dot{M}$. Interestingly, however, at least $\gamma$ Ara (and maybe HD 47240 - if its $\sin i$ were 0.5 ) are located at the 'right' position and might be worth being investigated in detail, e.g., by means of interferometry (see Chesneau, this volume) in combination with 2-D NLTE modeling (Georgiev et al. 2006). The outcome of such investigations will be of particular relevance for stellar evolution with rapid rotation, especially in the early Universe (see Ekström, this volume).

\section{Mass loss}

As we have seen, rapidly rotating B-supergiants are ideal test beds to check a number of theoretical predictions. Unfortunately, however, there exists only few such objects, since there is a rapid drop of rotation below $T_{\text {eff }} \approx 20 \mathrm{kK}$, as is obvious from the distribution of $v \sin i$ (e.g., Howarth et al. 1997). In a recent letter, Vink et al. (2010) tried to explain this finding based on two alternative scenarios (see also Langer, this volume). In scenario $I$, the low rotation rates of B-supergiants are suggested to be caused by braking due to an increased mass loss for $T_{\text {eff }}<25 \mathrm{kK}$, where this increased mass loss should be due to the so-called bi-stability jump. Vink et al. termed this process 'bi-stability braking'.

\subsection{The bi-stability jump}

The bi-stability jump itself has often been discussed and referred to in the literature, and goes back to findings by Pauldrach \& Puls (1990) when modeling the wind of P Cygni. These findings were generalized by Vink et al. $(2000,2001)$ in their work on stellar wind models for OB-stars: In the intermediate/late O-star and early B-star regime, the major contribution of driving lines in the lower wind (which are responsible for initiating the mass-loss rate) is from Fe IV. Below $T_{\text {eff }}=23 \mathrm{kK}$, however, Fe IV recombines more or less abruptly to Fe III. Since Fe IIIhas more effective lines than Fe IV, $N_{\text {eff }}$ increases (in parallel with a decrease of $\alpha$, see also Puls et al. 2000), which leads to an increase in $\dot{M}$ and a decrease in the terminal velocity, $v_{\infty}$. This is the theoretical basis for the $\kappa$-effect discussed in Sect. 2. Vink et al. (2000) predict a typical increase in $\dot{M}$ by a factor of 


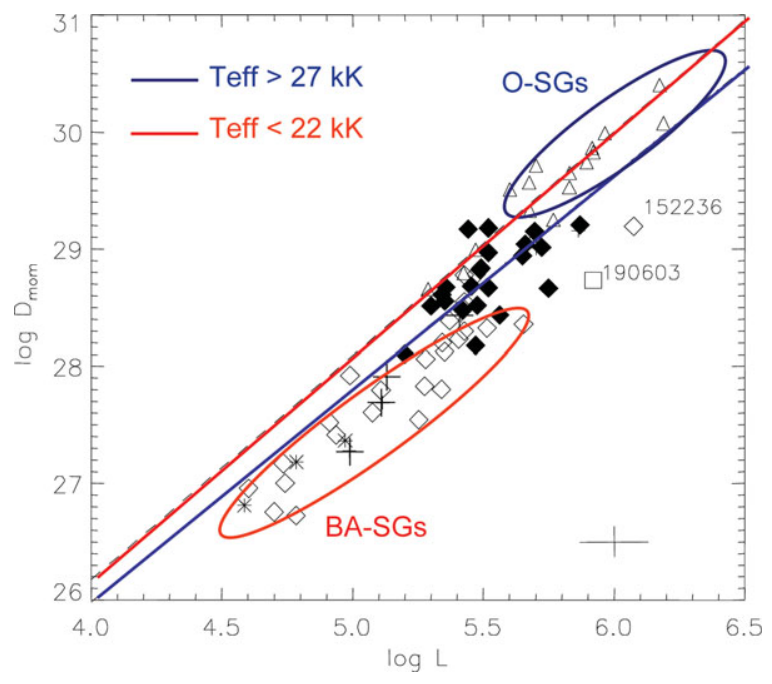

Figure 6. Modified wind-momenta for Galactic O- and B-supergiants. Dashed (red) and solid (blue) lines represent the predictions by Vink et al. (2000) for objects below and above $23 \mathrm{kK}$, respectively. Triangles are O-supergiants $\left(T_{\text {eff }}>27 \mathrm{kK}\right)$, filled diamonds early B-sgs with $T_{\text {eff }}>$ $22 \mathrm{kK}$ and open triangles B-supergiants below this value. The cross in the lower right displays the typical error bars. Adapted from Markova \& Puls (2008).

five, and a decrease in $v_{\infty}$ by a factor of two. Consequently, the wind-momenta from massive stars with $T_{\text {eff }}<23 \mathrm{kK}$ should be higher than those from stars of earlier spectral types (see dashed/solid lines in Fig. 6.) This important prediction needs to be checked observationally, not least because present day evolutionary codes often incorporate the corresponding 'mass-loss recipe'.

\subsection{Test beds for the bi-stability jump (I): B[e]-supergiants?}

The hybrid spectrum of B[e] supergiants can be explained by a two-component wind, with an outflowing 'disk' (equatorial wind) of low velocity, high density and low ionization, and a high velocity, low density and highly ionized polar wind (Zickgraf et al. 1986, 1989). A first explanation of this wind structure was given by Lamers \& Pauldrach (1991), who combined the effects of fast rotation and bi-stability jump, following the calculations from Pauldrach \& Puls (1990) for the latter: The high values of $g_{\text {eff }}$ together with the high ionization at the pole ( $T_{\text {eff }}$ calculated via von Zeipel) then give rise to a fast and thin polar wind, whereas the low $g_{\text {eff }} / T_{\text {eff }}$ values at the equator induce a slow and dense wind. (Note that $v_{\infty}$ scales with the photospheric escape velocity and thus with $g_{\text {eff }}$, see below).

Until now, the B[e]-sg mechanism is heavily debated. Owocki et al. (1998) pointed out that Lamers \& Pauldrach (1991), though accounting for gravity darkening when calculating the ionization, did not include its impact on $\dot{M}$ (Eq. 3.1). When gravity darkening is included into the accelerating flux, $F(\theta) \propto g_{\text {eff }}(\theta)$, a 'disk' formation becomes almost impossible, due to the counteracting effects of bi-stability and increased polar flux (see also Puls et al. 2008 and references therein). Simulations by Pelupessy et al. (2000), on the other hand, indicated that the bi-stability mechanism can work even when consistently accounting for gravity darkening, at least for a density contrast up until ten (observed values are on the order of hundred). Curé et al. (2005) showed that near critical rotation enables the wind to 'switch' from the standard, fast-accelerating solution to a slow, shallow-accelerating velocity law. This, in combination with the bi-stability effect, 
can lead to the formation of a slow and dense equatorial wind. Madura et al. (2007), finally, confirmed and explained the 'Curé-effect', but argued that gravity darkening is still a problem when aiming at a significant density contrast.

\subsection{Test beds for the bi-stability jump (II): 'normal' B-supergiants}

Thus, it is still unclear whether $\mathrm{B}[\mathrm{e}]$ supergiants can be used to verify the bi-stability effect. Consequently, we now consider 'normal' B-supergiants. One of the predictions by Vink et al. (2000) is an abrupt decrease of $v_{\infty}$ (see also Lamers et al. 1995) around the bistability jump (around $23 \mathrm{kK}$ ). Let us first consider this effect. Standard line-driven wind theory predicts that $v_{\infty} \approx 2.24 \alpha /(1-\alpha) v_{\text {esc }}$ (e.g., Puls et al. 1996), and a compilation of different measurements/analyses (mostly based on Evans et al. 2004 and Crowther et al. 2006) by Markova \& Puls (2008) shows that the average ratio $v_{\infty} / v_{\text {esc }} \approx 3.3$ for $T_{\text {eff }}$ $>23 \mathrm{kK}$ and $v_{\infty} / v_{\text {esc }} \approx 1.3$ for $T_{\text {eff }}<18 \mathrm{kK}$, with a gradual decrease in between (see also the original work by Evans et al. 2004; Crowther et al. 2006). Thus, there is an effect on $v_{\infty}$, but we also have to check the behaviour of the mass-loss rates. Conventionally, this is done by plotting the modified wind-momentum rate, $D_{\text {mom }}$, as a function of the stellar luminosity, since one of the major predictions from radiation driven wind theory is the well-known wind-momentum luminosity relation (WLR, Kudritzki et al. 1995),

$$
\log D_{\mathrm{mom}}=\log \left(\dot{M} v_{\infty}\left(R_{\star} / R_{\odot}\right)^{1 / 2}\right) \approx x \log \left(L / L_{\odot}\right)+\operatorname{offset}(\text { spect. type, metallicity) }
$$

where $x$ has a similar dependence as the offset. (Theoretically, $x=(\alpha-\delta)^{-1}$, where $\delta \approx 0.1$ accounts for ionization effects.)

Fig. 6 compares observationally inferred modified wind-momentum rates for OB-supergiants with the predictions from Vink et al. (2000) (for details, see Markova \& Puls 2008). As pointed out above, the predicted WLR for B-stars lies above the one for O-stars (more increase in $\dot{M}$ than decrease in $v_{\infty}$ ), whereas the observations show the opposite. The observed O-star rates (triangles, encircled in blue) lie above the predictions, which can be explained by clumping effects (see below), whereas the observed B-star rates for $T_{\text {eff }}$ $<22 \mathrm{kK}$ lie well below the predictions and those for $T_{\text {eff }}>22 \mathrm{kK}$ just connect the O-star regime and the cooler B-stars. With respect to $\dot{M}$ itself, a careful analysis shows that $\dot{M}$ either decreases in concert with $v_{\infty}$ (more likely), or at least remains unaffected (less likely). Globally, however, we do not see the predicted increase in $\dot{M}$, though a certain maximum around the location of the jump might be present (Benaglia et al. 2007). Thus, at least below the bi-stability jump there is a severe problem. Either the predicted $\dot{M}$ for cooler objects are too high, or the 'observed' (i.e., derived) ones are too low. Accounting for the observed O-star rates, the latter seems unlikely (and the inclusion of clumping would even increase the discrepancy for the B-stars). A way out of the dilemma might be the potential impact of the 'slow' wind solution (see above) on BA-supergiants, as suggested by Granada et al. (this volume).

\subsection{A separate population?}

Returning to the problem of the low rotation rates of B-supergiants and accounting for the above dilemma, one has to admit that if indeed the mass-loss rates were not increasing at the bi-stability jump, then there would be no bi-stability braking, and the rapid drop of rotation below $T_{\text {eff }}=20 \mathrm{kK}$ still needs to be explained. To this end, Vink et al. (2010) discuss an alternative scenario II (see also Langer, this volume): The cooler, slowly rotating supergiants might form an entirely separate, non core hydrogen-burning population, e.g., they might be products of binary evolution (though this is not generally expected to lead to slowly rotating stars), or they might be post-RSG or blue-loop stars.

Support of this second scenario is the finding that the majority of the cooler objects 
BI237: NIII4097

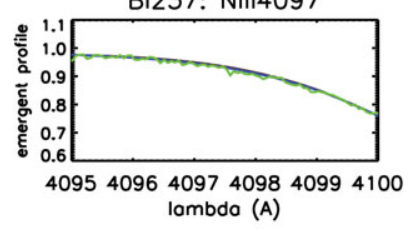

BI237: NIII4640/42

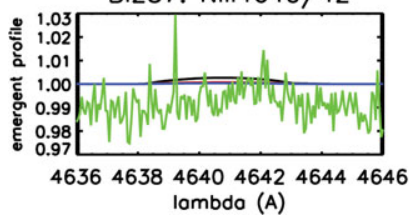

NGC346\#7: NIII4634
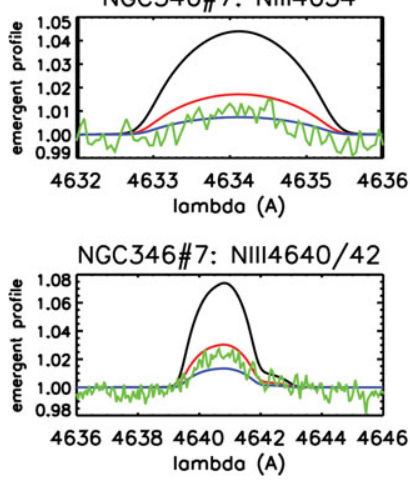

BI237: NIV4058

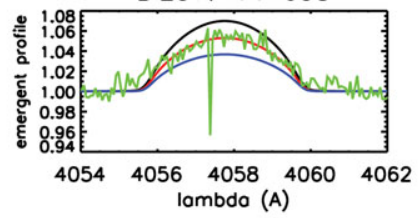

BI237: NIV6380

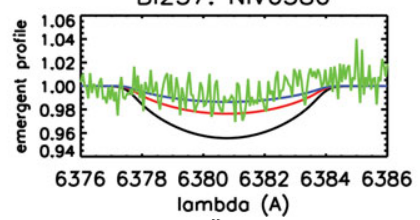

NGC346\#7: NIV4058

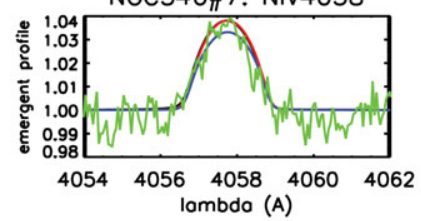

NGC346\#7: NIV6380

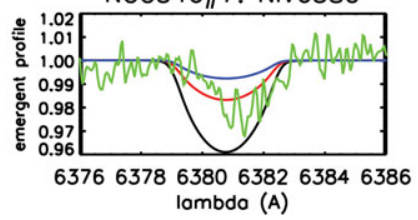

BI237: NV4603

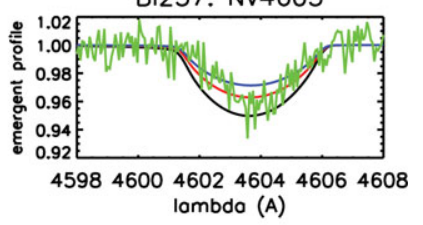

NGC346\#7: NV4603

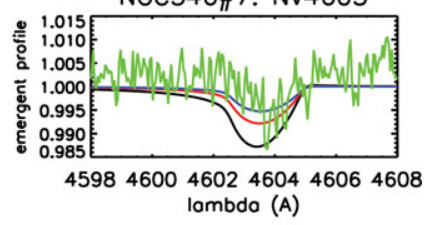

Figure 7. Strategic Nitrogen line profiles in the optical from ionization stages III to $\mathrm{V}$ for two early O-type stars. Observations in green, solid lines are synthetic profiles (calculated by the atomospheric code FASTwind, Puls et al. 2005) for different abundances: $\mathrm{N} / \mathrm{N}_{\odot}=0.2$ (blue), 0.4 (red) and 1.0 (black). Upper panels: BI237 $\left(\mathrm{O} 2 \mathrm{~V}\left(\left(\mathrm{f}^{*}\right)\right)\right.$ in the $\left.\mathrm{LMC}\right)$, with $T_{\text {eff }}=52 \mathrm{kK}$ and $\log g$ $=4.0$. The derived Nitrogen abundance is $\mathrm{N} / \mathrm{N}_{\odot}=0.4$ or $[\mathrm{N} / \mathrm{H}]=7.38$. Lower panels: NGC\#7 $\left(\mathrm{O} 4 \mathrm{~V}\left(\left(\mathrm{f}^{+}\right)\right)\right.$in the $\left.\mathrm{SMC}\right)$, with $T_{\text {eff }}=45 \mathrm{kK}$ and $\log g=4.0$. The derived Nitrogen abundance is $\mathrm{N} / \mathrm{N}_{\odot} \approx 0.2 \ldots 0.4$ or $[\mathrm{N} / \mathrm{H}] \approx 7.08 \ldots 7.38$.

(here: in the LMC) is strongly Nitrogen-enriched, which was one of the outcomes of the VLT-FLAMES survey of massive stars (Brott, this volume; see also Evans et al. 2008 for a brief summary of the project). Vink et al. argue that "although rotating models can in principle account for large $\mathrm{N}$ abundances, the fact that such a large number of the cooler objects is found to be N enriched suggests an evolved nature for these stars."

\subsection{Nitrogen abundances from O-stars}

So far, Nitrogen abundances could be derived only for a subset of the VLT-FLAMES sample stars, and corresponding data are missing particularly for the most massive and hottest stars. Indeed, when inspecting the available literature for massive stars, one realizes that metallic abundances, in particular of Nitrogen, which is the key element to check evolutionary predictions, are scarcely found for O-type stars. The simple reason is that they are difficult to determine, since the formation of NIII/IV lines (and lines from similar ions of $\mathrm{C}$ and $\mathrm{O}$ ) is problematic due to the impact of various processes that are absent or negligible at cooler spectral types, e.g., dielectronic recombination, mass-loss, and clumping. Within the VLT-FLAMES project, progress is under way (Rivero-González 2010), and in Fig. 7 we show two examples of N-abundance determinations for two early type O-stars in the LMC and SMC. Though no detailed comparison with evolutionary models has 
been made yet, the derived abundances for both objects are consistent, within the errorbars, with the average abundances from corresponding B-type stars of early evolutionary stages, which are $[\mathrm{N} / \mathrm{H}]=7.13 \pm 0.29$ for the $\mathrm{LMC}$ and $[\mathrm{N} / \mathrm{H}]=7.24 \pm 0.31$ for the SMC, respectively (Hunter et al. 2009).

\subsection{Wind clumping}

Mass loss is pivotal for the evolution/fate of massive stars (e.g., the formation of GRBs critically depends on the loss of angular momentum due to mass loss, see Ekström and Langer, this volume), their energy release, and their stellar yields. Thus, reliable mass-loss rates are urgently required (ideally better than a factor of two, Meynet et al. 1994). O-star mass-loss rates derived from the optical/radio have been found to be higher than predicted by the widely used mass-loss recipe from Vink et al. (2000) (see Fig. 6). The present hypothesis assumes that this discrepancy is due to neglected wind-clumping (small scale density inhomogeneities), originating from the line-driven instability, which results in overestimated mass-loss rates when using recombination-based diagnostics (Puls et al. 2008, and references therein). To check and infer the effects due to optically thin and thick clumps, and due to porosity in velocity space, on the various diagnostics, Sundqvist et al. $(2010,2011)$ have used the well observed star $\lambda$ Cep (O6I) as a test bed to derive a mass-loss rate of $1.5 \cdot 10^{-6} \mathrm{M}_{\odot} / \mathrm{yr}$. This is a factor of four lower than corresponding 'unclumped' values and a factor of two lower than the predictions by Vink et al. (2000).

\section{Very brief summary and conclusions}

We discussed OB-stars as extreme condition test beds, regarding effects due to pulsations, rapid rotation, and mass-loss. Rapidly rotating B-supergiants (though scarce) are particularly well suited to check a number of theoretical predictions, and the B1 supergiant $\gamma$ Ara may be a prime candidate for future diagnostics.

\section{Acknowledgements}

The authors gratefully acknowledge a travel grant from the IAU and the local organizers of this conference (J.P.), a grant from the IMPRS, Garching (J.O.S.), and a research grant from the German DFG (J.G.R.G.).

\section{References}

Aerts, C., Puls, J., Godart, M., \& Dupret, M.-A. 2009, A $\mathcal{E} A$ 508, 409

Aerts, C., Lefever, K., Baglin, A., Degroote, P. et al. 2010, A\&A (Letters), 513, L11

Benaglia, P., Vink, J. S., Martí, J., Maíz Apellániz, J. et al. 2007, A\&\&A 467, 1265

Bouret, J.-C., Lanz, T., \& Hillier, D. J. 2005, A\& $A$ 438, 301

Castor, J. I., Abbott, D. C., \& Klein, R. I. 1975, ApJ, 195, 157

Cranmer, S. R. \& Owocki, S. P. 1995, ApJ, 440, 308

Crowther, P. A., Lennon, D. J., \& Walborn, N. R. 2006, A\&A, 446, 279

Curé, M., Rial, D. F., \& Cidale, L. 2005, A\&\&A, 437, 929

Domiciano de Souza, A., Kervella, P., Jankov, S., Abe, L. et al. 2003, A\& A (Letters), 407, L47

Evans, C. J., Lennon, D. J., Trundle, C., Heap, S. R. et al. 2004, ApJ, 607, 451

Evans, C., Hunter, I., Smartt, S., Lennon, D. et al. 2008, The Messenger, 131, 25

Feldmeier, A., Kudritzki, R.-P., Palsa, R., Pauldrach, A. W. A. et al. 1997, A\&A, 320, 899

Feldmeier, A., Puls, J., \& Pauldrach, A. W. A. 1997, A\& A, 322, 878

Fullerton, A. W., Gies, D. R., \& Bolton, C. T. 1996, ApJS, 103, 475

Georgiev, L. N., Hillier, D. J., \& Zsargó, J. 2006, A\&A, 458, 597

Glatzel, W. 1998, A\&SA (Letters), 339, L5

Howarth, I. D., Siebert, K. W., Hussain, G. A. J., \& Prinja, R. K. 1997, MNRAS, 284, 265 
Hunter, I., Brott, I., Langer, N., Lennon, D. J. et al. 2009, A\& A, 496, 841

Kaufer, A., Stahl, O., Prinja, R. K., \& Witherick, D. 2006, A\&AA, 447, 325

Kudritzki, R.-P., Lennon, D. J., \& Puls, J. 1995, in: J.R. Walsh \& I.J. Danziger (eds.), Science with the VLT, Proc. ESO Workshop (Berlin: Springer), p. 246

Kervella, P. \& Domiciano de Souza, A. 2006, A\&A, 453, 1059

Lamers, H. J. G. \& Pauldrach, A. W. A. 1991, A\&A (Letters), 244, L5

Lamers, H. J. G. L. M., Snow, T. P., \& Lindholm, D. M. 1995, ApJ, 455, 269

Langer, N., Heger, A., \& Fliegner, J. 1997, in: T. R. Bedding, A. J. Booth, \& J. Davis (eds.), Fundamental stellar properties: The interaction between observation and theory, IAU Symposium 189, p. 343

Lefever, K., Puls, J., \& Aerts, C. 2007, A\&ऽA, 463, 1093

Maeder, A. 1999, A\&A, 347, 185

Maeder, A. \& Meynet, G. 2000, A\&A, 361, 159

Madura, T. I., Owocki, S. P., \& Feldmeier, A. 2007, ApJ, 660, 687

Markova, N. \& Puls, J. 2008, A\&SA, 478, 823

Massa, D., Fullerton, A. W., Nichols, J. S., Owocki, S. P. et al. 1995, ApJ (Letters) 452, L53

Meilland, A., Stee, P., Vannier, M., Millour, F. et al. 2007, A $\& A 464,59$

Meynet, G., Maeder, A., Schaller, G., Schaerer, D. et al. 1994, A\&SAS, 103, 97

Owocki, S. P., Cranmer, S. R., \& Gayley, K. G. 1998, in: A. M. Hubert \& C. Jaschek (eds.), $B[e]$ stars, Astrophysics and Space Science Library 233, p. 205

Pamyatnykh, A. A. 1999, AcA, 49, 119

Pauldrach, A. W. A. \& Puls, J. 1990, A\&A A, 237, 409

Pelupessy, I., Lamers, H. J. G. L. M., \& Vink, J. S. 2000, A\&A, 359, 695

Petrenz, P. \& Puls, J. 1996, A\&A, 312, 195

Petrenz, P. \& Puls, J. 2000, A\&A, 358, 956

Prinja, R. K., Massa, D., Fullerton, A. W., Howarth, I. D. et al. 1997, A\&GA, 318, 157

Puls, J., Kudritzki, R.-P., Herrero, A., Pauldrach, A. W. A. et al. 1996, A\&A, 305, 171

Puls, J., Springmann, U. \& Lennon, M. 2000, A\&AS, 141, 23

Puls, J., Urbaneja, M. A., Venero, R., Repolust, T. et al. 2005, A\&A A, 435, 669

Puls, J., Markova, N., Scuderi, S., Stanghellini, C. et al. 2006, A\&A, 454, 625

Puls, J., Vink, J. S., \& Najarro, F. 2008, A\&AR, 16, 209

Repolust, T., Puls, J., \& Herrero, A. 2004, A\&A A, 415, 349

Rivero-González, J. 2010, Diploma-Thesis (LMU München)

Saio, H., Kuschnig, R., Gautschy, A., Cameron, C. et al. 2006, ApJ, 650, 1111

Simón-Díaz, S., Herrero, A., Uytterhoeven, K., Castro, N. et al. 2010, ApJ (Letters) 720, 174

Sundqvist, J. O., Puls, J., \& Feldmeier, A. 2010, A\&A, 510A, 11

Sundqvist, J. O., Puls, J., Owocki, S., et al. 2011, in: P. Williams \& G. Rauw (eds.), The multiwavelength view of Hot, Massive Stars, $39^{\text {th }}$ Liège International Astrophysical Colloquium, in press

van Belle, G. T. 2010, in: T. Rivinius \& M. Curé (eds.), The Interferometric View on Hot Stars, Rev. Mexicana AyA Conference Series 38, p. 119

von Zeipel, H. 1924, MNRAS, 84, 665

Vink, J. S., de Koter, A., \& Lamers, H. J. G. L. M. 2000, $A \mathscr{G} A$, 362, 295

Vink, J. S., de Koter, A., \& Lamers, H. J. G. L. M. 2001, $A \mathscr{E} A, 369,574$

Vink, J. S., Davies, B., Harries, T. J., Oudmaijer, R. D. et al. 2009, A\&AA, 505, 743

Vink, J. S., Brott, I., Gräfener, G., Langer, N. et al. 2010, $A \& A$ (Letters), 512, L7

Waelkens, C., Aerts, C., Kestens, E., Grenon, M. et al. 1998, A\& A, 330, 215

Wende, S., Glatzel, W., \& Schuh, S. 2008, in: A. Werner \& T. Rauch (eds.), Hydrogen-Deficient Stars, ASP-CS 391, p. 319

Zahn, J.-P. 1992, A\&A, 265, 115

Zickgraf, F.-J., Wolf, B., Leitherer, C., Appenzeller, I. et al. 1986, A\&A, 163, 119

Zickgraf, F.-J., Wolf, B., Stahl, O., \& Humphreys, R. M. 1989, A\&̊A, 220, 206 